\title{
The amazing progress of high-power ultrafast thin-disk lasers
}

\author{
Clara J. Saraceno ${ }^{1 *}$ (D), Dirk Sutter ${ }^{2}$, Thomas Metzger $^{3}$ and Marwan Abdou Ahmed ${ }^{4}$
}

\begin{abstract}
Ultrafast lasers continue to be at the forefront of many scientific breakthroughs and technological achievements and progress in the performance of these systems continue to open doors in many new and exciting interdisciplinary fields. In particular, in the last decade, the average power of ultrafast lasers has seen a significant increase, opening up exciting new perspectives. Among the different technologies that have shaped these advances, thin-disk lasers have generated particularly spectacular breakthroughs. We review here the latest state-of-the-art of the technology and highlight new application fields of these cutting-edge laser systems.
\end{abstract}

Keywords: High-power lasers, Ultrafast lasers, Thin-disk lasers

\section{Introduction}

For several decades, Ti:Sapphire (Ti:Sa) laser technology has been the main workhorse of ultrafast laser science. Commercially available Ti:Sa multi-stage laser amplifiers routinely provide energetic $\mathrm{mJ}$-class pulses in the nearinfrared (NIR) with durations of few tens of femtoseconds and gigawatts to petawatts peak powers. As a gain material, Ti:Sa offers significant advantages for ultrafast lasers: a very large and broadband emission crosssection and good thermal conductivity make it an excellent candidate for the direct generation and amplification of ultrashort pulses. However, high saturation power and high doping levels are not possible while maintaining a good crystal quality, therefore longitudinally pumped bulk crystalline media in combination with small pump spots are typically required to achieve efficient laser operation. The poor heat extraction in this (bulk) geometry, combined with a large quantum defect, strongly limits the average power achievable from these systems to a few tens of watts in best cases, even with cryogenic cooling. As a result, typical Ti:Sa amplifier systems with high energy operate at repetition rates of few hundreds of $\mathrm{Hz}$ to few $\mathrm{kHz}$; while on the other hand, Ti: Sa mode-locked oscillators typically operating at tens of megahertz repetition rate remain at very low pulse

\footnotetext{
* Correspondence: clara.saraceno@ruhr-uni-bochum.de

'Photonics and Ultrafast Laser Science, Ruhr Universität Bochum, Bochum, Germany

Full list of author information is available at the end of the article
}

energy (in the nanojoule range). In many areas of science and technology, this compromise is a strong limitation or, in some cases, even a showstopper.

The emergence of new laser technologies based on $\mathrm{Yb}$ : doped gain materials in geometries that are more advantageous for heat extraction (fibers, slabs, thin-disks) has made it possible to overcome this unwanted compromise, and ultrafast laser systems based on all these architectures have now long surpassed the kilowatt average power milestone as depicted in Fig. 1 [1-5]. This new performance regime has not only allowed to overcome the actual limitations in many existing fields, but also has opened the door to many new applications. In the following, we place the focus of the present review paper on one ultrafast laser technology where progress in the last decade has been particularly remarkable - ultrafast thin-disk lasers (TDLs).

\section{State-of-the-art}

The thin-disk laser geometry

The TDL concept dates back to 1994 and was invented by A. Giesen and coworkers [6] at the Institut für Strahlwerkzeuge (IFSW) of the University of Stuttgart, Germany. In a TDL, the gain medium is shaped like a very thin (typically in the range of 100 to $300 \mu \mathrm{m}$ ) disk with a large diameter in comparison to its thickness (Fig. 2). The back side of the disk is coated with a highly reflecting mirror for both the pump and laser wavelengths and contacted on an appropriate 


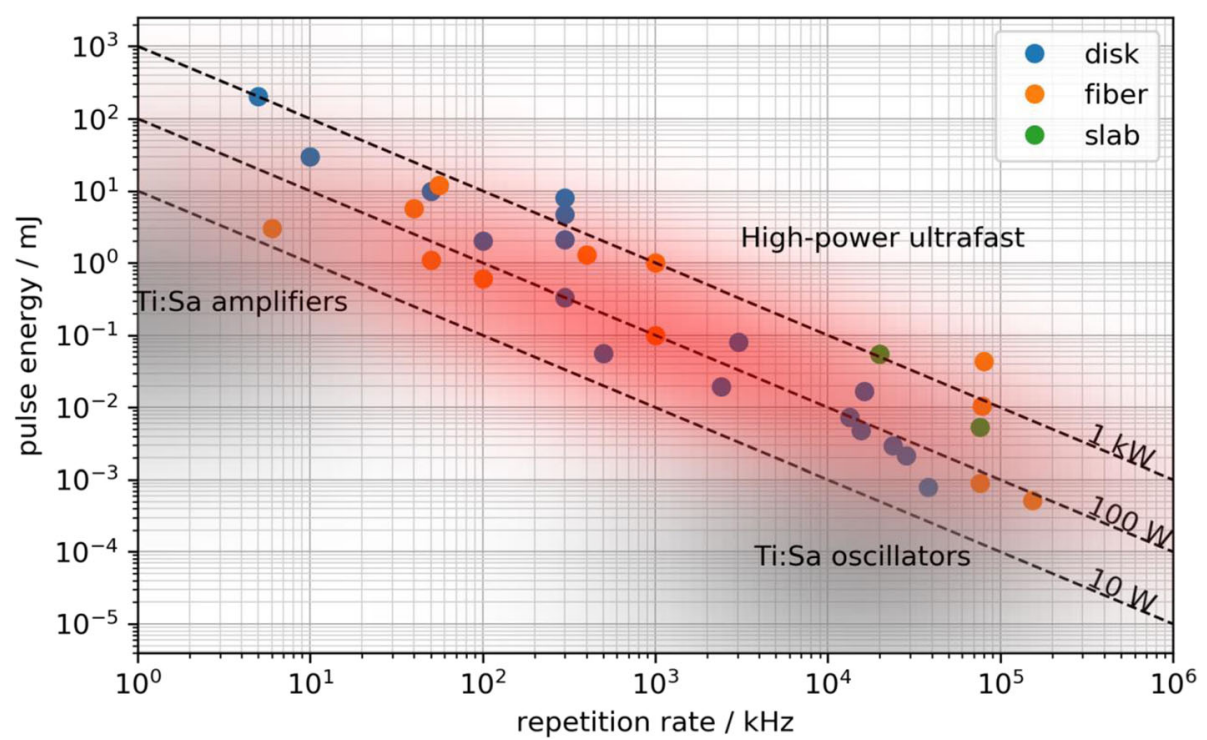

Fig. 1 Pulse energy versus repetition rate of representative state-of-the-art ultrafast lasers based on different geometries, both amplifiers and oscillators

heatsink (in latest results most commonly diamond). The front side is anti-reflection coated for the same wavelengths. In this way, the gain medium can be very efficiently water-cooled through the backside and used in a resonator or amplifier in reflection (as an "active mirror"). If the pump and laser spot sizes applied are correspondingly large (typically one to two orders of magnitude larger than the thickness), a one-dimensional heat-flow is obtained, and the scaling of the average output power can simply be achieved by increasing the pumped area and pump power by the same factor. Because of the short length of the gain medium, a correspondingly small single-pass absorption and gain are typically achieved in this geometry: both efficient pumping of solid-state laser media and amplification with significant gain in this geometry therefore require multiple-passes through the gain medium. For pumping, this is achieved by the implementation of an optical system composed of a parabolic mirror and prism-retro-reflectors that allow re-imaging of the pump beam on the thin-disk as many times as required to obtain sufficient pumplight absorption [7]. Commercially available industrial pumping modules with up to 72 pump-light passes provide the necessary platform for efficient pumping in this geometry. For efficient amplification, multiple passes are achieved either in a resonator (oscillator or regenerative amplifier) or by geometric multiplexing (like the pump pass arrangement as depicted in Fig. 2 a) or a combination of both.

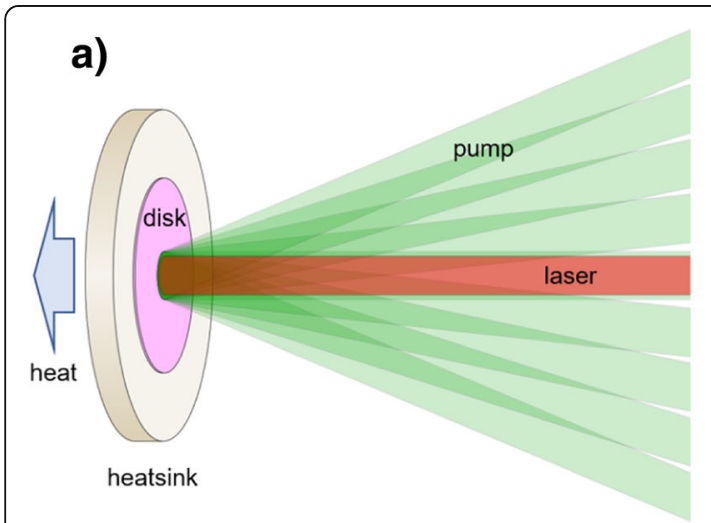

\section{b)}

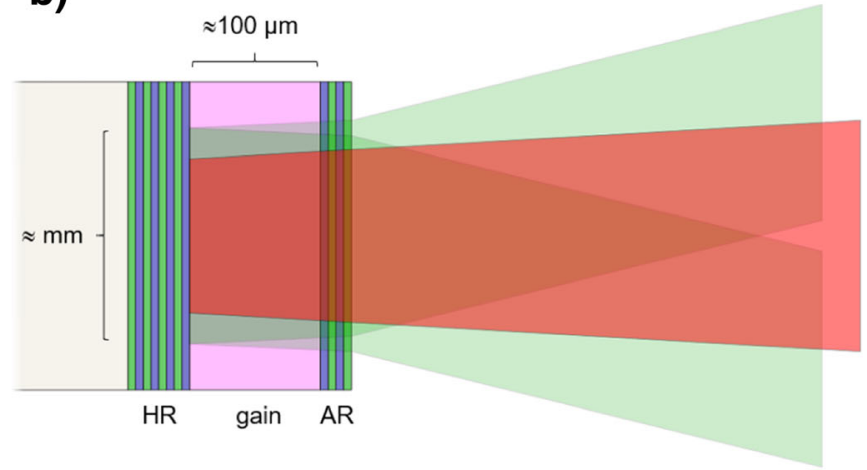

Fig. 2 TDL concept a) Schematic illustration of one of the most typically used arrangement (to scale of a typical Yb:YAG TDL): multi-pass pumping and single-pass gain. The re-imaged pump beam after each disk pass can be thought of as multiple beams impingent on the disk with decreasing intensity at each bounce. A similar multi-pass arrangement can be made for the laser beam $\mathbf{b}$ ) schematic side view of the disk (not to scale) 
In addition to an excellent heat extraction, the thindisk geometry offers significant advantages for ultrafast operation. The large mode areas on the gain medium and the short propagation distance of the pulses through the gain medium make it inherently advantageous for small nonlinearities at very high pulse energies. TDL regenerative and multi-pass amplifiers have proven to be extremely successful to scale energy and average output power of ultrafast laser systems to new frontiers, exceeding the kilowatt average power with pulse energies raging from the multi-millijoule to the multi-hundreds of millijoule level $[3,4]$. The extremely high pulse energies achievable stand in strong contrast to what is achievable with kilowatt-class chirped fiber amplifiers. Additionally, the TDL geometry offers the unique possibility of direct mode-locking of oscillators with very high average power operating in the multi- $\mathrm{MHz}$ repetition rate with tens of microjoules of pulse energy. This again stands in strong contrast with other geometries such as fibers and slabs, where high-power oscillators are difficult to power and energy scale, due to the long interaction length between the pulses and the gain medium. Since their first demonstration in the year 2000 [8], mode-locked thin-disk oscillators have consistently achieved orders of magnitude higher average power and pulse energy than any other ultrafast oscillator technology, reaching comparable levels to advanced high-power amplifiers operating at $\mathrm{MHz}$ repetition rate [9].

\section{Ultrafast TDL systems}

In Fig. 3, we present schematically the different concepts nowadays used for the generation and amplification of ultrashort pulses in the thin-disk geometry, with hundreds of watts to kilowatts of average power. Most state-of-the-art results have been achieved using $\mathrm{Yb}$ : doped garnets, due to their small quantum defect and excellent thermo-mechanical properties, sufficient bandwidth for femtosecond operation, and well-established manufacturing perfection in the TDL geometry. We therefore focus our discussion on these laser systems.

In a thin-disk multi-pass amplifier, the core components are the thin-disk itself and the array of mirrors to allow the geometrical folding of the seed beam though the disk. Figure 3-a shows a schematic of such an amplifier. The amplifier medium consists of an anti-reflection coated, plane-parallel Yb:YAG (or Yb:LuAG) thin-disk (typically with a radius of curvature of $>20 \mathrm{~m}$, a thickness of $\sim 100 \mu \mathrm{m})$ mounted on a diamond heat sink. The beam path is folded over the disk by an array of 40 to 80 plane mirrors, allowing for a total gain of one to two orders of magnitude. To avoid tight foci along the beam propagation in the amplifier, a collimated seed beam is used. At sufficient seed energy, amplified pulse energies approaching the Joule-level have been demonstrated [10].

In a thin-disk regenerative amplifier, multiple passes through the gain medium are achieved by placing the disk in a resonator with a Pockels cell, which controls the number of roundtrips and ejects strongly amplified pulses after many roundtrips (Fig. 3-b). For particularly high-energy, chirped-pulse amplification (CPA) may be employed, i.e. the seed pulses may be temporally stretched to ns durations, typically in a fiber Bragg grating, and a larger beam-diameter pulse compressor can be used for the amplified beam, allowing for multiGW peak powers. However, in contrast to fiber (i.e. waveguide-confined) lasers, intra-cavity nonlinearities can be avoided by scaling the transverse beam size at many orders of magnitude higher peak powers, such that CPA can often be avoided in thin-disk regenerative amplifiers.

In modelocked thin-disk oscillators (Fig. 3-c), a singletransverse mode, high-power laser oscillator is modelocked to generate ultrashort pulses. These systems are
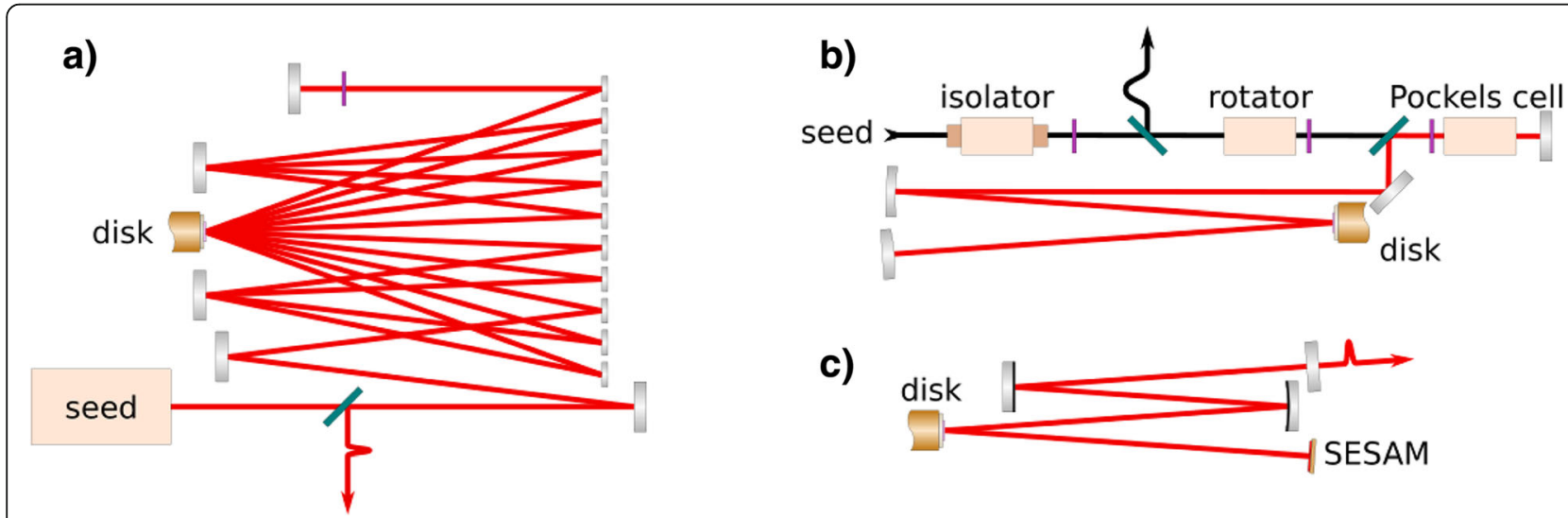

Fig. 3 Schematic illustration of the different schemes for the generation of ultrashort pulses with hundreds of watts to kilowatts with thin-disk lasers. a) multi-pass amplifier b) regenerative amplifier c) modelocked oscillator (here illustrated is the case of SESAM modelocking) 
very attractive, because they provide energetic pulses at $\mathrm{MHz}$ repetition rate from a compact, single oscillator, and they may even give access to increased intra-cavity peak powers. Most commonly, Semiconductor Saturable Absorber Mirror (SESAM) [11] soliton-modelocking $[12,13]$ or Kerr-Lens modelocking [14] are used for pulse formation, and nonlinearity and dispersion must balance each other at each roundtrip of the pulses throughout the resonator. In this case, it is particularly challenging to achieve robust single-mode operation, stable modelocked pulses at extremely high intracavity average and peak powers, free of damage of intracavity optics. Other modelocking mechanisms in the weak positive dispersion regime have been attempted $[15,16]$, but so far with limited success. A promising route currently being explored is the use of modelocking regimes which are more tolerant to strong nonlinearities, as commonly exploited in fiber lasers [17], but proof-of-principle experiments remain so far undemonstrated.

\section{Most recent state of the art}

Thin-disk regenerative amplifiers [18, 19] have progressed particularly fast. Since their introduction as the world's first high average power industrial ultrafast lasers with $50 \mathrm{~W}$ of average power back in 2007 they have become the most successful commercial ultrafast lasers for direct micromachining [20]. The first high power industrial femtosecond lasers exceeding $100 \mathrm{~W}$, demonstrated in 2013, were based on a CPA thin-disk regenerative amplifier, and obtained gigawatt peak powers [21]. Such peak powers are even possible in CPA-free systems using cm-size Pockels cells [22]. Most recent results of CPA based thin-disk regenerative amplifiers have demonstrated $200 \mathrm{~mJ}$ of pulse energy at $1 \mathrm{~kW}$ of average power in 1-ps long-pulses (i.e. approaching TW peak powers) at $5 \mathrm{kHz}$ repetition rate [3]. Progress is underway to reach the J-level at $\mathrm{kW}$ average power by using several thin-disk multi-pass amplifiers in series [10]. Thindisk multi-pass amplifiers used as booster amplifiers of medium power regenerative thin-disk or fiber-bulk amplifiers has also seen spectacular progress, evolving from systems capable of delivering very high pulse energy at moderate average power [23] to $\mathrm{kW}$ level amplifiers with very large parameter flexibility $[4,24,25]$. The latest experiments led to the extraction of up to $2.45 \mathrm{~kW}$ at a repetition rate of $300 \mathrm{kHz}$, corresponding to a pulse energy of more than $8 \mathrm{~mJ}$, and a pulse duration below 8-ps [26]. Very recently, an average output power of $>1 \mathrm{~kW}$ with ultrashort pulses of a duration of $\sim 500 \mathrm{fs}$, and a pulse energy of $1 \mathrm{~mJ}$ have been achieved in the context of the project Hiperdias [27].

Modelocked TDLs are very promising for applications benefiting from very high repetition rates in the multi-
$\mathrm{MHz}$ regime. Average powers up to $275 \mathrm{~W}$ with $600 \mathrm{fs}$ long pulses at $17 \mathrm{MHz}$, corresponding to a peak power of $26 \mathrm{MW}$ [28], and pulse energies up to $80 \mu \mathrm{J}$ with 1-ps pulses (66 MW of peak power) [29] have been demonstrated with SESAM modelocking. Further scaling to $350 \mathrm{~W}$ of average power has most recently been announced [30]. Comparable average powers and peak powers have also been demonstrated with Kerr-lens modelocking, where $270 \mathrm{~W}$ with $330 \mathrm{fs}$ pulse duration (38 MW peak power) have been reached [31] and more recently, $150 \mathrm{~W}$ with $150 \mathrm{fs}$ pulses (60 MW peak power) [32]. Naturally, Kerr-lens modelocking achieves shorter pulse durations, although a compromise between output power and pulse duration also needs to be met. Many of these results were achieved by operating the oscillator in vacuum chambers $[28,29,32]$ to minimize the detrimental nonlinearity of air, which affects modelocking stability at highest intracavity peak power. Alternatively, the intracavity power can be reduced by multiplying the number of gain passes inside the modelocked resonator. This approach (also referred to as "active multi-pass cell") was demonstrated early on using SESAM modelocking, and impressive results were achieved [33], reaching $40 \mu \mathrm{J}$ pulse energy without the need for vacuum.

\section{Applications}

Much of the progress achieved in the development of these powerful ultrafast laser systems has been driven by the growing industrial market of ultrafast lasers for high-precision material processing. Cold ablation of a large variety of materials can be driven at higher speeds with high repetition rate, high energy ultrafast lasers. In fact, many of the above-mentioned state-of-the-art laser systems have been demonstrated in an industrial $R \& D$ environment. In this field, the advantages of $\mathrm{kW}$ ultrafast lasers are starting to be demonstrated: for example, efficient processing of carbon fiber reinforced polymer (CFRP) with negligible thermal damage was demonstrated, which was enabled by the application of a thindisk multipass amplifiers system that generates ps pulses at an average output power exceeding $1 \mathrm{~kW}$ [34]. More recently, very high productivity surface structuring of steel for antibacterial applications has been demonstrated using the same amplifier system [35]. Additionally, new findings appear to show that glass cutting can still be scaled significantly further [36].

In scientific research, many efforts to increase the average power of NIR ultrafast TDLs have aimed at increasing the flux of XUV pulses obtained via high harmonic generation [37]. Large progress in this area has been achieved with high-power ultrafast fiber amplifiers [38], however, significant progress is also expected in the near future with TDL driving sources, in 
particular at much high energy levels, as the availability of shorter pulses keeps making progress. This will benefit a large number of time-resolved experimental methods using XUV pulses, which could be dramatically improved with higher flux [39]. One example is the study of ionization dynamics using coincidence measurements, where long recording times are necessary to reach reasonable statistics. In this case, a higher repetition rate allows to significantly reduce measurement times and/or improve signal-to-noise ratio. Another example is the study of photoemission dynamics from surfaces, where space charge effects limit the applicable pulse intensity, therefore a high repetition rate is desired to reach sufficiently high signal-to-noise ratio at lower excitation intensity.

In parallel to the large progress achieved in abovementioned areas, other applications areas are nowadays emerging that benefit from the availability of ultrafast laser sources with ever-increasing average power. Several large research consortia have been established in the last years aiming to explore the new possibilities offered by these laser systems: the laser lightning rod project which aims to use filaments to divert lightning [http://llr-fet.eu/], the FemtoSurf project to explore multi-beam processing [https://cordis.europa.eu/project/rcn/219130/factsheet/en], the $\mathrm{kW}$-Flexiburst project to explore next generation processing at $\mathrm{kW}$ average power and $\mathrm{GHz}$ repetition rates [https://cordis.europa.eu/project/rcn/ $219108 /$ factsheet/en], the LAMPAS project aiming to explore large scale surface functionalization [https://cordis.europa.eu/project/rcn/219130/en], and the Hiperdias project to explore processing of $\mathrm{Si}$ and Diamond at $\mathrm{kW}$ class femtosecond laser pulses [www.hiperdias.eu], to cite only a few examples.

It is important to notice that many new areas will also emerge as these laser systems are explored as driving sources for further nonlinear conversion. In general, there is a clear trend toward extending high-power operation to a wide spectral range ranging all the way from the XUV [40] to the terahertz $(\mathrm{THz})$ regime [41], going through the deep-UV and the mid and far-IR ranges [42].

\section{Conclusion}

The enormous application potential of these novel sources is only now starting to bloom, going together with their increasing commercial availability. We believe that in the near future, many new applications will emerge. All these systems are far from reaching their fundamental limitations: we expect that they will continue breaking frontiers in terms of pulse energy, average power and peak power in the next few years. Further average power scaling will most likely require multiplexing the number of gain amplification elements to spread the thermal load over several disks, such as is already the case in the $\mathrm{kW}$ class regenerative amplifier. Ongoing progress in various components for extremely highpower levels (optics, dispersive elements, etc.) will be key in these achievements.

One important challenge for use of these sources in scientific applications remains to extend current power and energy records to significantly shorter pulse durations. One attractive path to achieve this is using novel broadband gain materials to generate short pulses directly. All record-holding ultrafast laser systems mentioned above are based on the relatively narrowband gain material Yb:YAG, which poses many challenges to achieve sub-100 fs pulse durations with highest power and energies. Finding new gain material capable of supporting the current performance levels of Yb:YAG while providing more broadband amplification remains one challenge in the field, both with oscillators and amplifiers. Whereas many other more broadband Yb-doped materials have been tested in the thin-disk geometry, mostly in modelocked operation in the goal of obtaining short pulses [43-47], most results remain in the proofof-principle state with moderate average powers $<30 \mathrm{~W}$ and/or low pulse energies $<2 \mu$, due to the low quality of novel gain crystals and additional difficulties related to the generation of very short pulses. Ongoing efforts to improve the quality of this and other Yb-doped broadband gain materials are expected to bring further improvements to the achievable levels. In parallel to these efforts, large progress has been made in finding paths for efficient external pulse compression of these high-average power laser systems using hollow-core fibers [48], multi-pass cells [49-52], hybrid setups including multi-plate compressors [53], and even spectral broadening in the amplifier itself [54] bringing these applications one step closer. Additionally, as the pulse durations from these systems keeps getting shorter and approach the few-cycle regime, carrier-envelope phase control becomes increasingly relevant, in particular for high-power oscillators which can be used as powerful seeds for booster amplifiers $[55,56]$.

More recently, laser materials based on other dopant ions have started to be explored for the TDL geometry. The first proof-of-principle demonstration of a Ti:Sa laser in the thin-disk geometry was achieved most recently [57]. Whereas the latest obtained results still remain moderate for the thin-disk geometry (13 W) [58], this represents an important first milestone in the potential direct generation of high-power few-cycle pulses at high average power. Furthermore, external pulse compression obviously allows for reaching few-fs pulse durations even starting from the (sub-) picosecond regime $[51,52]$. Finally, extending the wavelength coverage available from TDLs to longer wavelengths also 
represents a very promising research direction for ultrafast thin-disk oscillators and amplifiers, which would expand the application possibilities of these sources even further. Recently, a first Kerr-lens modelocked oscillator based on Ho:YAG has been demonstrated with $25 \mathrm{~W}$, $270 \mathrm{fs}$ [59] and further reports on CW operation with record-high power $(50 \mathrm{~W}$ for Ho:YAG and $24 \mathrm{~W}$ for Tm: YAG, [60]) illustrate the potential of this emerging area.

\section{Acknowledgements}

None.

\section{Authors' contributions}

All authors contributed equally in compiling this review. All authors read and approved the final manuscript.

\section{Funding}

Clara J. Saraceno acknowledges funding by the Alexander von Humboldt Foundation, the German Research Council (Deutsche Forschungsgemeinschaft, DFG) in the framework of CRC/TRR196 (MARIE) for project C10 and by the Deutsche Forschungsgemeinschaft (DFG, German Research Foundation) under Germany's Excellence Strategy - EXC-2033 - Projektnumber 390677874. Marwan Abdou Ahmed acknowledges support from the Project Tresclean, which is an initiative of the Photonics Public Private Partnership and has received funding from the European Union's Horizon 2020 research and innovation programme under grant agreement No 687613, the Project Hiperdias, which is an initiative of the Photonics Public Private Partnership and has received funding from the European Union's Horizon 2020 research and innovation programme under grant agreement No 687880 and the Project RAZipol, which has received funding from the European Union's Seventh Framework Programme for research, technological development and demonstration under grant agreement no. 619237 and the project TiSaTD which has received funding from the European Union's Seventh Framework Programme for research, technological development and demonstration under grant agreement no. 619177.

TRUMPF Laser GmbH acknowledges support of the ultrafast kW disk laser research by the German Federal Ministry of Education and Research BMBF in the project ScULPT (Scaling Ultrafast Laser Productive Precision Processing Technology), which is an EffiLAS (Efficient High-Power Laser Sources) initiative and has received partial funding under contract 13 N13927, and from the Project LAMpAS, which is an initiative of the Photonics Public Private Partnership and has received funding from the European Union's Horizon 2020 research and innovation programme under grant agreement No. 825132.

TRUMPF Scientific Lasers GmbH + Co. KG acknowledges partial funding from the Project LLR, which is a Future and Emerging Technologies (FET) Open initiative and has received funding the European Union's Horizon 2020 research and innovation programme under grant agreement No. 737033.

\section{Availability of data and materials}

Upon request to the authors.

\section{Competing interests}

The authors declare that they have no competing interests.

\section{Author details}

${ }^{1}$ Photonics and Ultrafast Laser Science, Ruhr Universität Bochum, Bochum, Germany. ${ }^{2}$ TRUMPF Lasers, Schramberg, Germany. ${ }^{3}$ TRUMPF Scientific Lasers, Unterföhring, Germany. ${ }^{4}$ Institut für Strahlwerkzeuge (IFSW), University of Stuttgart, Stuttgart, Germany.

Received: 4 March 2019 Accepted: 20 May 2019

Published online: 19 June 2019

\section{References}

1. Russbueldt, P., Mans, T., Weitenberg, J., Hoffmann, H.D., Poprawe, R.: Compact diode-pumped 1.1 kW Yb:YAG Innoslab femtosecond amplifier. Opt. Lett. 35, 4169-4171 (2010)
2. Gaida, C., Gebhardt, M., Heuermann, T., Stutzki, F., Jauregui, C., Limpert, J.: Ultrafast thulium fiber laser system emitting more than $1 \mathrm{~kW}$ of average power. Opt. Lett. 43, 5853-5856 (2018)

3. Nubbemeyer, T., Kaumanns, M., Ueffing, M., Gorjan, M., Alismail, A., Fattahi,

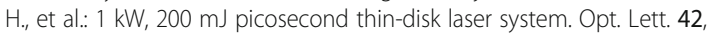
1381-1384 (2017)

4. Negel, J.P., Loescher, A., Voss, A., Bauer, D., Sutter, D., Killi, A., et al.: Ultrafast thin-disk multipass laser amplifier delivering $1.4 \mathrm{~kW}(4.7 \mathrm{~mJ}, 1030 \mathrm{~nm})$ average power converted to $820 \mathrm{~W}$ at $515 \mathrm{~nm}$ and $234 \mathrm{~W}$ at $343 \mathrm{~nm}$. Opt Express. 23, 21064-21077 (2015)

5. Müller, M., Klenke, A., Steinkopff, A., Stark, H., Tünnermann, A., Limpert, J.: 3.5 kW coherently combined ultrafast fiber laser. Opt. Lett. 43, 6037-6040 (2018)

6. Giesen, A., Hügel, H., Voss, A., Wittig, K., Brauch, U., Opower, H.: Scalable concept for diode-pumped high-power solid-state lasers. Appl. Phys. B Lasers Opt. 58, 365-372 (1994)

7. Erhard, S.: Pumpoptiken und Resonatoren für den Scheibenlaser. University of Stuttgart (2002)

8. Aus der Au, J., Spühler, G.J., Südmeyer, T., Paschotta, R., Hövel, R., Moser, M., et al.: 16.2 W average power from a diode-pumped femtosecond Yb:YAG thin disk laser. Opt. Lett. 25, 859-861 (2000)

9. Saraceno, C.J., Emaury, F., Schriber, C., Diebold, A., Hoffmann, M., Golling, M., et al.: Toward Millijoule-level high-power ultrafast thin-disk oscillators. IEEE J. Sel. Top. Quantum Electron. 21, (2015)

10. Herkommer, C., Krötz, P., Klingebiel, S., Wandt, C., Bauer, D., Michel, K., et al.: Towards a Joule-Class Ultrafast Thin-Disk Based Amplifier at Kilohertz Repetition Rate. Presented at the Conference on Lasers and Electro-Optics, San Jose, California (2019)

11. Keller, U., Weingarten, K.J., Kärtner, F.X., Kopf, D., Braun, B., Jung, I.D., et al.: Semiconductor saturable absorber mirrors (SESAMs) for femtosecond to nanosecond pulse generation in solid-state lasers. IEEE J. Sel. Top. Quantum Electron. 2, 435-453 (1996)

12. Kärtner, F.X., Keller, U.: Stabilization of soliton-like pulses with a slow saturable absorber. Opt. Lett. 20, 16-18 (1995)

13. Paschotta, R., Keller, U.: Passive mode locking with slow saturable absorbers. Appl. Phys. B Lasers Opt. 73, 653-662 (2001)

14. Brabec, T., Spielmann, C., Curley, P.F., Krausz, F.: Kerr lens mode locking. Opt. Lett. 17, 1292-1294 (1992)

15. Palmer, G., Schultze, M., Siegel, M., Emons, M., Bünting, U., Morgner, U. Passively mode-locked $\mathrm{Yb}: \mathrm{KLu}\left(\mathrm{WO}_{4}\right)_{2}$ thin-disk oscillator operated in the positive and negative dispersion regime. Opt. Lett. 33, 1608-1610 (2008)

16. Pronin, O., Brons, J., Grasse, C., Pervak, V., Boehm, G., Amann, M.C., et al.: High-power Kerr-lens mode-locked Yb:YAG thin-disk oscillator in the positive dispersion regime. Opt. Lett. 37, 3543-3545 (2012)

17. Ilday, F.O., Kesim, D.K., Hoffmann, M., Saraceno, C.J.: Discrete Similariton and dissipative soliton Modelocking for energy scaling ultrafast thin-disk laser oscillators. IEEE J. Sel. Top. Quantum Electron. 24, 1-2 (2018)

18. Hönninger, C., Johannsen, I., Moser, M., Zhang, G., Giesen, A., Keller, U. Diode-pumped thin disk Yb:YAG regenerative amplifier. Appl. Phys. B Lasers Opt. 65, 423-426 (1997)

19. Metzger, T., Schwarz, A., Teisset, C.Y., Sutter, D., Killi, A., Kienberger, R., et al.: High-repetition-rate picosecond pump laser based on a Yb:YAG disk amplifier for optical parametric amplification. Opt. Lett. 34, 2123-2125 (2009)

20. Heckl, O.H., Kleinbauer, J., Bauer, D., Weiler, S., Metzger, T., Sutter, D.H.: Ultrafast thin-disk lasers. In: Nolte, S., Schrempel, F., Dausinger, F. (eds.) Ultrashort Pulse Laser Technology: Laser Sources and Applications, pp. 93 115. Springer International Publishing, Cham (2016)

21. Fleischhaker, R., Gebs, R., Budnicki, A., Wolf, M., Kleinbauer, J., Sutter, D.H. Compact gigawatt-class sub-picosecond Yb: YAG thin-disk regenerative chirped-pulse amplifier with high average power at up to $800 \mathrm{kHz}$. Presented at the Conference on and International Quantum Electronics Conference Lasers and Electro-Optics Europe (Cleo Europe) (2013)

22. Ueffing, M., Lange, R., Pleyer, T., Pervak, V., Metzger, T., Sutter, D., et al.: Direct regenerative amplification of femtosecond pulses to the multimillijoule level. Opt. Lett. 41, 3840-3843 (2016)

23. Antognini, A., Schuhmann, K., Amaro, F.D., Biraben, F., Dax, A., Giesen, A., et al.: Thin-disk Yb:YAG oscillator-amplifier laser, ASE, and effective Yb:YAG lifetime. IEEE J. Quantum Electron. 45, 983-995 (2009)

24. Negel, J.P., Voss, A., Ahmed, M.A., Bauer, D., Sutter, D., Killi, A., et al.: 1.1 kW average output power from a thin-disk multipass amplifier for ultrashort laser pulses. Opt. Lett. 38, 5442-5445 (2013) 
25. Negel, J.P., Loescher, A., Dannecker, B., Oldorf, P., Reichel, S., Peters, R., et al. Thin-disk multipass amplifier for fs pulses delivering $400 \mathrm{~W}$ of average and $2.0 \mathrm{GW}$ of peak power for linear polarization as well as $235 \mathrm{~W}$ and $1.2 \mathrm{GW}$ for radial polarization. Appl. Phys. B Lasers Opt. 123, 156 (2017)

26. Abdou-Ahmed, M.: Development of High-Power Thin-Disk Lasers: Status and Perspectives. Presented at the JNPLI, Strasbourg (2017)

27. Röcker, C., Loescher, A., Negel, J.P., Delaigue, M., Morin, F., Hönninger, C., et al.: kW-class thin-disk multipass amplifier delivering sub-300fs pulses at a repetition rate of $1 \mathrm{MHz}$ for high-throughput laser-based material processing, p. 2018. In International Congress on Applications of Lasers \& Electro-Optics (ICALEO), Orlando, Florida

28. Saraceno, C.J., Emaury, F., Heckl, O.H., Baer, C.R.E., Hoffmann, M., Schriber, C., et al.: $275 \mathrm{~W}$ average output power from a femtosecond thin disk oscillator operated in a vacuum environment. Opt. Express. 20, 23535-23541 (2012)

29. Saraceno, C.J., Emaury, F., Schriber, C., Hoffmann, M., Golling, M., Südmeyer, T., et al.: Ultrafast thin-disk laser with $80 \mu \mathrm{J}$ pulse energy and $242 \mathrm{~W}$ of average power. Opt. Lett. 39, 9-12 (2014)

30. Saltarelli, F., Graumann, I., Lang, L., Bauer, D., Phillips, C., Keller, U.: Power Scaling of Ultrafast Laser Oscillators: 350-W Output Power Sub-Ps SESAMModelocked Thin-Disk Laser. Presented at the Conference on Lasers and Electro Optics CLEO 2019, San Jose, CA (2019)

31. Brons, J., Pervak, V., Fedulova, E., Bauer, D., Sutter, D., Kalashnikov, V.L., et al.: Energy scaling of Kerr-lens mode-locked thin-disk oscillators. Opt. Lett. 39, 6442-6445 (2014)

32. Brons, J., Pervak, V., Bauer, D., Sutter, D., Pronin, O., Krausz, F.: Powerful 100-fs-scale Kerr-lens mode-locked thin-disk oscillator. Opt. Lett. 41, 3567-3570 (2016)

33. Bauer, D., Zawischa, I., Sutter, D.H., Killi, A., Dekorsy, T.: Mode-locked Yb: YAG thin-disk oscillator with $41 \mu \mathrm{J}$ pulse energy at $145 \mathrm{~W}$ average infrared power and high power frequency conversion. Opt. Express. 20, 9698-9704 (2012)

34. Graf, T., Weber, R., Abdou-Ahmed, M., Onuseit, V., Freitag, C., Weidenmann, M., et al.: Efficient High-Quality Processing of CFRP with a kW Ultrafast ThinDisk Laser. Presented at the Advanced Solid State Lasers (2015)

35. Faas, S., Weber, R., Graf, T.: Heat accumulation controlled surface functionalization of stainless steel with structuring rates up to $500 \mathrm{~mm} 2 / \mathrm{s}$. Procedia CIRP. 74, 324-327 (2018)

36. Jenne, M., Flamm, D., Ouaj, T., Hellstern, J., Kleiner, J., Grossmann, D., et al. High-quality tailored-edge cleaving using aberration-corrected Bessel-like beams. Opt. Lett. 43, 3164-3167 (2018)

37. Emaury, F., Diebold, A., Saraceno, C.J., Keller, U.: Compact extreme ultraviolet source at megahertz pulse repetition rate with a low-noise ultrafast thindisk laser oscillator. Optica. 2, 980-984 (2015)

38. Hädrich, S., Klenke, A., Rothhardt, J., Krebs, M., Hoffmann, A., Pronin, O., et al.: High photon flux table-top coherent extreme-ultraviolet source. Nat Photon. 8, 779-783 (2014)

39. Leone, S.R., McCurdy, C.W., Burgdoerfer, J., Cederbaum, L.S., Chang, Z. Dudovich, N., et al.: What will it take to observe processes in 'real time'? Nat. Phot. 8, 162-166 (2014)

40. Südmeyer, T., Marchese, S.V., Hashimoto, S., Baer, C.R.E., Gingras, G., Witzel, B. et al.: Femtosecond laser oscillators for high-field science. Nat. Phot. 2, 599604 (2008)

41. Meyer, F., Hekmat, N., Mansourzadeh, S., Fobbe, F., Aslani, F., Hoffmann, M., et al.: Optical rectification of a $100 \mathrm{~W}$ average power mode-locked thin-disk oscillator. Opt. Lett. 43, 5909-5912 (2018)

42. Seidel, M., Xiao, X., Hussain, S., Arisholm, G., Hartung, A., Zawilski, K.T., et al.: Multi-watt, multi-octave, mid-infrared femtosecond source. Sci. Adv. 4, eaaq1526 (2018)

43. Südmeyer, T., Kränkel, C., Baer, C.R.E., Heckl, O.H., Saraceno, C.J., Golling, M., et al.: High-power ultrafast thin disk laser oscillators and their potential for sub-100-femtosecond pulse generation. Appl. Phys. B Lasers Opt. 97. 281-295 (2009)

44. Beil, K., Saraceno, C.J., Schriber, C., Emaury, F., Heckl, O.H., Baer, C.R.E., et al.: $\mathrm{Yb}$-doped mixed sesquioxides for ultrashort pulse generation in the thin disk laser setup. Appl. Phys. B Lasers Opt. 113, 13-18 (2013)

45. Diebold, A., Emaury, F., Saraceno, C.J., Schriber, C., Golling, M., Südmeyer, T., et al.: 62-fs pulses from a SESAM modelocked Yb:CALGO thin disk laser. Opt. Lett. 38, 3842-3845 (2013)

46. Modsching, N., Paradis, C., Labaye, F., Gaponenko, M., Graumann, I.J., Diebold, A., et al.: Kerr lens mode-locked Yb:CALGO thin-disk laser. Opt. Lett. 43, 879-882 (2018)
47. Modsching, N., Drs, J., Fischer, J., Paradis, C., Labaye, F., Gaponenko, M., et al.: 21 W Average Power Sub-100-Fs Yb:Lu2O3 Thin-Disk Laser. Presented at the Conference on Lasers and Electro-Optics, San Jose, California (2019)

48. Emaury, F., Saraceno, C.J., Debord, B., Ghosh, D., Diebold, A., Gerome, F., et al.: Efficient spectral broadening in the 100-W average power regime using gas-filled kagome HC-PCF and pulse compression. Opt. Lett. 39, 6843-6846 (2014)

49. Schulte, J., Sartorius, T., Weitenberg, J., Vernaleken, A., Russbueldt, P.: Nonlinear pulse compression in a multi-pass cell. Opt. Lett. 41, 4511-4514 (2016)

50. Fritsch, K., Poetzlberger, M., Pervak, V., Brons, J., Pronin, O.: All-solid-state multipass spectral broadening to sub-20 fs. Opt. Lett. 43, 4643-4646 (2018)

51. Kaumanns, M., Pervak, V., Kormin, D., Leshchenko, V., Kessel, A., Ueffing, M., et al.: Multipass spectral broadening of $18 \mathrm{~mJ}$ pulses compressible from 1.3 ps to 41 fs. Opt. Lett. 43, 5877-5880 (2018)

52. Lavenu, L., Natile, M., Guichard, F., Délen, X., Hanna, M., Zaouter, Y., et al.: High-power two-cycle ultrafast source based on hybrid nonlinear compression. Opt. Express. 27, 1958-1967 (2019)

53. Tsai, C.-L., Meyer, F., Omar, A., Wang, Y., L. a, Y., Lu, C.-H., et al.: 27-Fs, 166MW Pulses at $98 \mathrm{~W}$ Average Power from Highly Efficient Thin-Disk Oscillator Driven Nonlinear Compressor. Presented at the Conference on Lasers and Electro-Optics, San Jose, California (2019)

54. Dannecker, B., Negel, J.P., Loescher, A., Oldorf, P., Reichel, S., Peters, R., et al.: Exploiting nonlinear spectral broadening in a $400 \mathrm{~W}$ Yb:YAG thin-disk multipass amplifier to achieve $2 \mathrm{~mJ}$ pulses with sub-150 fs duration. Opt. Commun. 429, 180-188 (2018)

55. Emaury, F., Diebold, A., Klenner, A., Saraceno, C.J., Schilt, S., Sudmeyer, T., et al.: Frequency comb offset dynamics of SESAM modelocked thin disk lasers. Opt. Express. 23, 21836-21856 (2015)

56. Grobmeyer, S., Brons, J., Seidel, M., Pronin, O.: Carrier-envelope-offset frequency stable $100 \mathrm{~W}$-level femtosecond thin-disk oscillator. Laser Photonics Rev. 13, 1800256 (2019)

57. Wolter, J.H., Ahmed, M.A., Graf, T.: Thin-disk laser operation of Ti:sapphire. Opt. Lett. 42, 1624-1627 (2017)

58. Wolter, A.V.J., Balmer, R., Ricaud, S., Antier, M., Simon-Boisson, C., Graf, T. Ahmed, M.A.: Symmetrically-Cooled Ti:Sapphire Thin-Disk Laser Using Single-Crystal Diamond Heat Spreaders. Presented at the Laser Congress (Advanced Solid State Lasers), Boston (2018)

59. Zhang, J.W., Mak, K.F., Pronin, O.: Kerr-Lens mode-locked 2-mu m thin-disk lasers. IEEE J. Sel. Top. Quantum Electron. 24, (2018)

60. Zhang, J., Schulze, F., Mak, K.F., Pervak, V., Bauer, D., Sutter, D., et al.: Highpower, high-efficiency tm:YAG and ho:YAG thin-disk lasers. Laser Photonics Rev. 12, (2018)

\section{Publisher's Note}

Springer Nature remains neutral with regard to jurisdictional claims in published maps and institutional affiliations.

\section{Submit your manuscript to a SpringerOpen ${ }^{\circ}$ journal and benefit from:}

- Convenient online submission

- Rigorous peer review

- Open access: articles freely available online

- High visibility within the field

- Retaining the copyright to your article

Submit your next manuscript at $>$ springeropen.com 